\title{
Energy Harvesting from the Secondary Resonances of a Nonlinear Piezoelectric Beam under Hard Harmonic Excitation
}

\author{
Masoud Rezaei ${ }^{1}$, Siamak E Khadem ${ }^{1 *}$, M. I. Friswell ${ }^{2}$ \\ ${ }^{1}$ Department of Mechanical Engineering, Tarbiat Modares University, Tehran, Iran \\ ${ }^{2}$ College of Engineering, Swansea University, Bay Campus, Fabian Way, Crymlyn Burrows, Swansea SA1 8EN, UK
}

\begin{abstract}
This paper investigates the dynamical response of a nonlinear piezoelectric (PZT) energy harvester under a hard harmonic excitation and assesses its output power. The system is composed of a unimorph cantilever beam with a tip mass and exposed to an harmonic tip excitation with a hard forcing amplitude. First, the governing dimensionless nonlinear electromechanical ordinary differential equations (ODEs) are obtained. Next, the multiple scales method (MSM) is exploited to provide an approximate-analytical solution for the ODEs in hard and soft forcing scenarios. It is observed that, the hard force results in suband super-harmonic resonances. The MSM-based solutions are then validated by a numerical integration method and a good agreement is observed between the approximate-analytical and numerical results. Furthermore, utilizing the MSM-based solutions for the subharmonic, superharmonic, and soft primary resonances cases, the associated frequency and force response curves are constructed. It is revealed that the hard excitation leads to a remarkable voltage generation in the secondary resonances; this leads to a broadband energy harvesting. In addition, the time-domain electrical responses of the secondary resonances are also obtained and compared with each other. Finally, the three-dimensional graphs of the electrical power versus detuning parameter and time constant ratio in the cases of the secondary resonances are plotted. The results show that the optimum output power of the superharmonic resonance is considerably larger than the maximum power of the subharmonic resonance case.
\end{abstract}

\section{KEYWORDS:}

PZT energy harvesting; Hard excitation; Subharmonic resonance; Superharmonic resonance; Broadband energy harvesting; Numerical verification

\section{*Professor and The Corresponding Author:}

S. E. Khadem, Department of Mechanical Engineering, Tarbiat Modares University, Tehran, Iran, P.O. Box 14115177

E-mail Address: khadem@modares.ac.ir

Tel/Fax: +98(21)82883388 


\section{1-Introduction}

The use of battery-reliant electronic devices with sub-milliwatt power consumption ranging from sensors, controllers, and medical implants to wearable electronics is rapidly growing. The typical electrochemical batteries used in these devices are unsatisfactory because of the high costs due to periodical replacement and chemical pollution. The search for portable, cheap, and renewable energy resources for these lowpower electronic devices has motivated the harvesting of small-scale energy from the environment [1]. Harnessing untapped ambient environmental energy and converting it to useful electrical energy offers an abundant source of sustainable and renewable energy. Potential sources for renewable-based electricity generation are wind, wave, sunlight, and kinetic energies. The integration of energy harvesters with these devices improves their efficiency by providing local and green energy sources enabling them to operate autonomously. Contrary to the other sources, kinetic energy has been shown to be a promising source due to its ubiquity and diversity. Accordingly, electrical energy can be harvested through vibrations [2-4], flow-induced instabilities [5-9], combined loadings [10], and so on. The wasted energy can be converted to electrical form with the aid of electrostatic [11, 12], electromagnetic [13-16], and piezoelectric [17-19] transducers. Although each of these mechanisms has their own benefits and inevitable drawbacks, piezoelectricity is pursued increasingly owing to the possibility of downsizing devices and operation over a wide range of frequencies.

Reviewing the vast research on piezoelectric vibration energy harvesters (PVEH), initial works have modelled simple PVEHs. Erturk and Inman [20,21] provided lumped and distributed parameter models of a linear beam based PVEH and presented analytical solutions for the deflection and voltage responses. Their experimental results revealed that both of the developed models can capture the dynamics of the electromechanical system, whereas the distributed-parameter model is more precise. However, the efficiency of the initial linear devices is hindered due to their narrow resonance region and they are illsuited for operating under environmental excitation, which mainly consist of time-varying frequencies. Hence, novel designs have been increasingly perused to cope with this challenge and capturing the channeled energy in an efficient way [22, 23]. Firstly, the idea of integrating active/passive tuning mechanisms was investigated to match the excitation and PVEH resonance frequencies [24-26]. However, this tuning adds to the complexity and decreases the efficiency of PVEHs [27]. Inspired by the positive role of inherent nonlinearities in the structure to broaden the resonance bandwidth of the system, the concept of the intentional application of nonlinear restoring forces emerged to compensate the mistuning [17]. Daqaq et al. [28] reviewed the techniques that inject nonlinearity through magnetic coupling and 
observed that, utilizing an external magnetic field, a mono/bistable cubic nonlinearity develops in the system and improves its efficiency through widening the resonance frequency bandwidth. This leads to superior performance of nonlinear energy harvesters over linear ones, and consequently nonlinearly designed PVEH are used extensively for efficient energy scavenging. Recently, Deng et al. [29] proposed a poly-stable PVEH using an array of piezoelectric beams with tip magnets. Empirical results showed that the operating bandwidth was widened by a factor of 41 , with respect to its single linear counterpart. Moreover, the voltage and output power density increased by $178 \%$ and $760 \%$, respectively, compared to the single linear harvester.

However, nonlinear energy harvesters can also experience secondary resonances under the external excitation depending on the forcing amplitude. Masana and Daqaq [30] investigated a bistable PVEH under longitudinal excitation, in the vicinity of its super-harmonic resonance of order two. The theoretical and experimental results showed that, at the specific forcing levels, the harvester can produce considerable power in the vicinity of the half of its fundamental frequency. Lin et al. [31] investigated a magnetically coupled PVEH operating over a broad range of frequencies. It was observed that the voltage of the proposed system was three, four, and five times that of the linear counterpart in the presence of the stochastic, subharmonic, and ultraharmonic resonances, respectively. Syta et al. [32] studied the response of a bistable piezoelectric energy harvester, composed of a vertical beam with a tip mass. The results revealed that, in addition to the excitation frequency, the harvester is capable of producing comparable voltages at subharmonics of $1 / 3$ and $2 / 3$. Huguet et al. [33] examined the response of a bistable energy harvester in subharmonic oscillations, both numerically and experimentally. It was shown that activating the subharmonic resonances broadens the operating frequency bandwidth of the energy harvester by $180 \%$ compared to the case of primary resonance. Panyam et al. [34] investigated energy harvesting from the subharmonic parametric excitation of a bistable PZT beam. It was observed that, depending on the amplitude and frequency of the external excitation, the system can experience periodic intra-well oscillations, intra- and inter-well chaotic vibrations, and periodic inter-well motions. Moreover, the occurrence of subharmonic resonance, can result in broadband energy harvesting. Huguet et al. [35] studied, both analytically and experimentally, the subharmonic behavior of a bistable PVEH. The results showed that the frequency bandwidth of the harvester was $25 \mathrm{~Hz}$ in the presence of a sole harmonic 1 resonance. However, by combining harmonic 1 and subharmonic 3 resonances, the bistable system could harness more than $100 \mu \mathrm{W}$ with a $70 \mathrm{~Hz}$ frequency bandwidth. This implies a $180 \%$ increase in the resonance bandwidth. Chen et al. [36] investigated a bistable micro/nano-scale plate energy harvester 
with a one-third super-harmonic resonance. It was observed that the presence of large amplitude vibrations in the super-harmonic region results in a $25 \mathrm{mV}$ amplification in the output voltage.

Based on the literature, the occurrence of super-harmonic resonances in a vibratory energy harvester, is proven to result in a remarkable increase in output voltage. However, the investigation by Nayfeh and Mook [37] revealed that a nonlinear oscillator can undergo both sub- and super-harmonic resonances in the presence of a sufficiently large forcing level, referred to hard excitation. Although these resonances have already been noticed in mechanical oscillators $[38,39]$, to the best of the authors' knowledge and from reviewing the literature, they have not been investigated analytically in the framework of energy harvesting. Consequently, there is not a comprehensive analytical study of the mechanical and electrical response along with the optimum electrical output of a PVEH in the presence of a hard excitation which leads to the sub- and super-harmonic resonances. The absence of such a study characterizing the response of a PVEH under hard excitation was also stated in Refs [33, 35]. To fill this gap, this work provides an analytical investigation to classify the probable resonances that a nonlinear PVEH can undergo in the presence of a hard external excitation, for the first time. Hence, the response of the system can be characterized over a wide range of frequencies and a bridge can be constructed between analytical, numerical, and empirical studies. Moreover, the optimum electrical power will be investigated to determine the efficiency of the PVEH at the secondary resonances. This allows the efficiency of a PVEH to be assessed for the secondary resonance cases. Hence, the dimensionless nonlinear electromechanical equations governing a cantilever unimorph carrying a tip mass under harmonic excitation are provided. Then, using the multiple scales method (MSM), the approximate-analytical solutions of the deflection and voltage for the secondary resonances are obtained. Finally, the MSM-based solutions, the numerical validation, and the optimum power are provided. The results show that the output power is maximum for the superharmonic resonance scenario.

\section{2-Mathematical Modelling}

Figure 1a shows the proposed system composed of a cantilever unimorph beam carrying a cubic tip mass and exposed to an harmonic tip excitation. The corresponding simplified electrical circuit is also illustrated in Fig. 1b. 


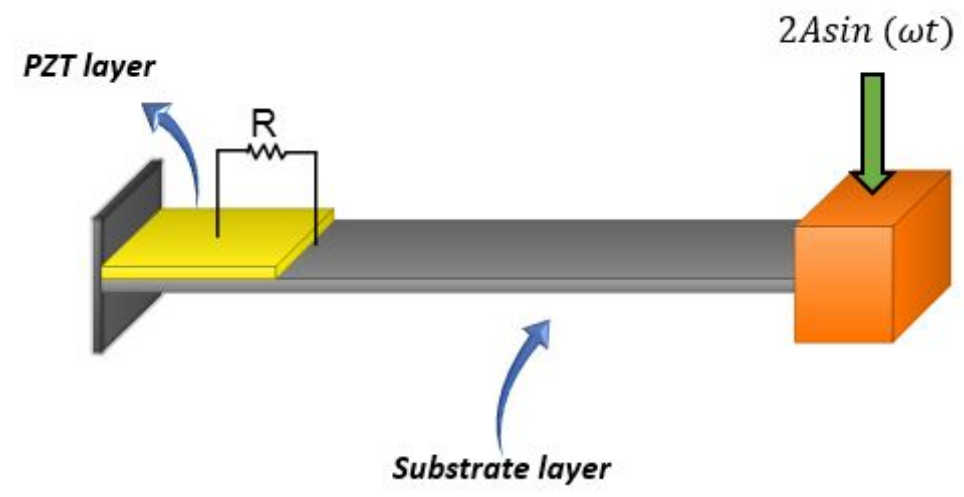

(a)

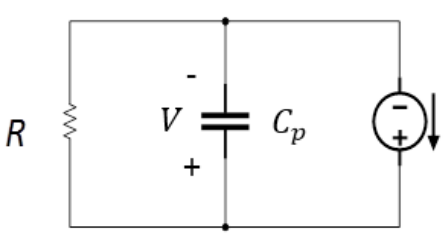

(b)

Fig. 1 (a) The schematic of the piezoelectric energy harvester and (b) a simplified electrical circuit

Utilizing the experimentally validated data in Ref. [40], the governing equations for a single mode approximation of the transverse displacement $(u)$ and generated voltage $(\bar{V})$ of the piezoelectric energy harvester with a cubic nonlinearity can be stated as:

$$
\begin{aligned}
& m \ddot{u}+C \dot{u}+k_{1} u+k_{3} u^{3}-\theta \bar{V}=2 A \cos (\omega \tau) \\
& C_{p} \dot{\bar{V}}+\frac{\bar{V}}{R}+\theta \dot{u}=0
\end{aligned}
$$

where $m, C, k_{1}$, and $k_{3}$ are, respectively, the mass, viscous damping, linear, and nonlinear stiffness coefficients of the piezoelectric beam. Moreover, $A$ and $\Omega$ denote the amplitude and frequency of the external excitation, respectively. In addition, $C_{p}$ is the piezoelectric capacitance, $R$ is the load resistance, and $\theta$ is the linear electromechanical coupling coefficient. In Eq. (1-a), $\tau$ is the time and the over-dot denotes differentiation with respect to time. To generalize the results, the dimensionless form of the ODEs (Eq. (1)) is obtained by defining the following dimensionless parameters:

$$
t=\sqrt{k_{1} / m} \tau ; x=\frac{u}{\tilde{L}} ; \quad v=\frac{C_{p}}{\widetilde{L} \Theta} \bar{V}
$$

where $t, x$, and $v$ represent the dimensionless time, displacement, and voltage, respectively. Moreover, $\tilde{L}$ is a length scale and here is assumed to be the height of the tip mass (h). The physical properties assumed for the examples that follow are listed in Table 1 [41]: 
Table 1. Physical properties of the energy harvester [41]

\begin{tabular}{lc}
\hline \hline \multicolumn{1}{c}{ Parameter } & Value \\
\hline \hline Effective mass $(m)$ & $0.1134 \mathrm{~kg}$ \\
Beam length $(L)$ & $209 \mathrm{~mm}$ \\
Beam width $(W)$ & $24 \mathrm{~mm}$ \\
Beam thickness $(\tilde{t})$ & $1 \mathrm{~mm}$ \\
Damping ratio $(\zeta)$ & 0.003 \\
Linear stiffness $\left(k_{1}\right)$ & $58.2 \mathrm{~N} / \mathrm{m}$ \\
Nonlinear stiffness $\left(k_{3}\right)$ & $1.2 \times 10^{3} \mathrm{~N} / \mathrm{m}^{3}$ \\
Capacitance of PZT layer $\left(C_{p}\right)$ & $187 \mathrm{nF}$ \\
Electromechanical coupling $(\Theta)$ & $1.9 \times 10^{-4} \mathrm{~N} / \mathrm{V}$ \\
Height of the tip mass $(h)$ & $5 \mathrm{~cm}$ \\
\hline \hline
\end{tabular}

Since both of the linear and nonlinear stiffness coefficients are positive, the elastic energy of the harvester is monostable. Using the dimensionless parameters, the nondimensional governing equations are obtained as:

$$
\begin{aligned}
& \ddot{x}+2 \zeta \dot{x}+x+\kappa x^{3}-\Theta v=2 f \cos (\Omega t) \\
& \dot{v}+\alpha v+\dot{x}=0
\end{aligned}
$$

where

$$
\zeta=\frac{C}{2 \sqrt{k_{1} / m}} ; \kappa=\frac{k_{3} h^{2}}{k_{1}} ; \Theta=\frac{\theta^{2}}{\mathrm{k}_{1} C_{p}} ; f=\frac{A}{k_{1} h} ; \Omega=\frac{\omega}{\sqrt{k_{1} / m}} ; \alpha=\frac{1}{R C_{p} \sqrt{k_{1} / m}}
$$

Now, the over-dot shows differentiation with respect to the dimensionless time. Moreover $\zeta, \kappa, \Theta, f$, and $\Omega$ are the dimensionless damping coefficient, cubic stiffness, electromechanical coupling, forcing amplitude, and excitation frequency, respectively. Furthermore, $R C_{p}$ is called the time constant of the electrical circuit [42] and $\alpha$ is the dimensionless time constant ratio.

\section{3- Approximate-Analytical Solution}

To characterize the dynamical response of the energy harvester, the multiple scales method (MSM) will be used to obtain the approximate-analytical solution of Eq. (3). To this end, the displacement and voltage responses of the harvester are expanded as [43]: 


$$
\begin{aligned}
& x(t ; \varepsilon)=x_{0}\left(T_{o}, T_{1}\right)+\varepsilon x_{1}\left(T_{0}, T_{1}\right)+\mathcal{O}\left(\varepsilon^{2}\right) \\
& v(t ; \varepsilon)=v_{0}\left(T_{o}, T_{1}\right)+\varepsilon v_{1}\left(T_{0}, T_{1}\right)+\mathcal{O}\left(\varepsilon^{2}\right)
\end{aligned}
$$

where $\varepsilon$ is a small bookkeeping parameter; $T_{0}=t$ and $T_{1}=\varepsilon t$ are fast and slow time scales, respectively. Accordingly, the time derivatives can be rewritten as [43]:

$$
\begin{aligned}
& \frac{d}{d t}=D_{0}+\varepsilon D_{1}+\mathcal{O}\left(\varepsilon^{2}\right) \\
& \frac{d^{2}}{d t^{2}}=D_{0}^{2}+2 \varepsilon D_{0} D_{1}+\mathcal{O}\left(\varepsilon^{2}\right)
\end{aligned}
$$

in which, $D_{n}=\partial / \partial T_{n}$. The dimensionless damping ratio, cubic nonlinearity, and electromechanical coupling are scaled such that they appear in the first-order of the perturbation problem $(\mathcal{O}(\varepsilon)$ ). Physically, the system is assumed to be lightly damped, and hence the damping term can be assumed first order. Similarly, the electromechanical coupling is first order, since efficient energy harvesting requires mechanical resonance and too much coupling will increase the effective damping in the system. In this system, we assume a weak nonlinearity and so this term is also first order. Thus, mathematically:

$$
\zeta=\varepsilon \zeta ; \kappa=\varepsilon \kappa ; \Theta=\varepsilon \Theta
$$

When the external excitation amplitude is hard $(f=\mathcal{O}(1))$, it must be scaled such that it appears in the zeroth-order $\left(\mathcal{O}\left(\varepsilon^{0}\right)\right)$ perturbation problem [37, 44]. Introducing Eqs. (5)-(7) in Eq. (3) and considering $f=$ $\mathcal{O}(1)$, one finds:

$$
\begin{aligned}
& \left(D_{0}^{2}+2 \varepsilon D_{0} D_{1}\right)\left(x_{0}+\varepsilon x_{1}\right)+2 \varepsilon \zeta\left(D_{0}+\varepsilon D_{1}\right)\left(x_{0}+\varepsilon x_{1}\right)+\left(x_{0}+\varepsilon x_{1}\right)+\kappa\left(x_{0}+\varepsilon x_{1}\right)^{3}- \\
& \Theta\left(v_{0}+\varepsilon v_{1}\right)=2 f \cos (\Omega t) \\
& \left(D_{0}+\varepsilon D_{1}\right)\left(v_{0}+\varepsilon v_{1}\right)+\alpha\left(v_{0}+\varepsilon v_{1}\right)+\left(D_{0}+\varepsilon D_{1}\right)\left(x_{0}+\varepsilon x_{1}\right)=0
\end{aligned}
$$

Separating $\mathcal{O}\left(\varepsilon^{0}\right)$ coefficients leads to:

$$
\left\{\begin{array}{l}
D_{0}^{2} x_{0}+x_{0}=f\left(e^{i \Omega t}+e^{-i \Omega t}\right) \\
D_{0} v_{0}+\alpha V_{0}+D_{0} x_{0}=0
\end{array}\right.
$$

The solution of the Eq. (9) is:

$$
x_{0}=A e^{i T_{0}}+\frac{f}{1-\Omega^{2}} e^{i \Omega T_{0}}+c c
$$


$v_{0}=-\left\{\frac{A i e^{i T_{0}}}{\alpha+i}+\frac{f \Omega e^{i \Omega T_{0}}}{\left(1-\Omega^{2}\right)(\alpha+i \Omega)} e^{i \Omega T_{0}}+c c\right\}$

where $A=A\left(T_{1}\right), F=f / 1-\Omega^{2}$, and $c c$ denotes the complex conjugate of the preceding terms. Moreover, separating $\mathcal{O}\left(\varepsilon^{1}\right)$ coefficients gives:

$$
\left\{\begin{array}{l}
D_{0}^{2} x_{1}+x_{1}=-2 D_{0} D_{1} x_{0}-2 \zeta D_{0} x_{0}+\Theta v_{o}-\kappa x_{0}^{3} \\
D_{0} v_{1}+\alpha v_{1}=-D_{1} V_{0}-\left\{D_{0} x_{0}+D_{1} x_{0}\right\}
\end{array}\right.
$$

Inserting the solution of the zeroth-order problem (Eq. (10)) in Eq. (11) yields:

$$
\begin{aligned}
& D_{0}^{2} x_{1}+x_{1}=-2\left\{A^{\prime} i e^{i T_{0}}+C C\right\}-2 \zeta\left\{A i e^{i T_{0}}+F i \Omega e^{i \Omega T_{0}}+c c\right\}-\Theta\left\{\frac{A i}{\alpha+i} e^{i T_{0}}+\frac{i F \Omega e^{i \Omega T_{0}}}{(\alpha+i \Omega)}+\right. \\
& c c\}-\kappa\left\{A^{3} e^{3 i T_{0}}+F^{3} e^{3 i \Omega T_{0}}+3 A^{2} \bar{A} e^{i T_{0}}+3 A^{2} F e^{i(2+\Omega) T_{0}}+3 A F^{2} e^{i(1+2 \Omega) T_{0}}+\right. \\
& \left.3 A^{2} F e^{i(2-\Omega) T_{0}}+3 A F^{2} e^{i(1-2 \Omega) T_{0}}+3 F^{3} e^{i \Omega T_{0}}+6 A \bar{A} F e^{i \Omega T_{0}}+6 A F^{2} e^{i T_{0}}+c c\right\}
\end{aligned}
$$

Inspecting Eq. (12), some of the terms are able to generate resonant behavior, the so-called secular terms. Separating the secular terms, the following equation is obtained:

$$
\begin{aligned}
& D_{0}^{2} x_{1}+x_{1}=-2\left\{A^{\prime} i e^{i T_{0}}+c c\right\}-2 \zeta\left\{A i e^{i T_{0}}+F i \Omega e^{i \Omega T_{0}}+c c\right\}-\Theta\left\{\frac{A i}{\alpha+i} e^{i T_{0}}+\frac{i F \Omega e^{i \Omega T_{0}}}{(\alpha+i \Omega)}+c c\right\}- \\
& \kappa\left\{3 A^{2} F e^{i(2-\Omega) T_{0}}+3 \bar{A}^{2} F e^{i(\Omega-2) T_{0}}+3 \bar{A} F^{2} e^{i(2 \Omega-1) T_{0}}+6 A \bar{A} F e^{i \Omega T_{0}}+F^{3} e^{3 i \Omega T_{0}}+3 F^{3} e^{i \Omega T_{0}}+\right. \\
& \left.3 A^{2} \bar{A} e^{i T_{0}}+6 A F^{2} e^{i T_{0}}+c c\right\}+\kappa\{N S T\}
\end{aligned}
$$

where NST denotes non-secular terms. Examining Eq. (13), it can be observed that, in addition to the terms proportional to $e^{i T_{0}}$, the terms involving $e^{i(\Omega-2) T_{0}}, e^{3 i \Omega T_{0}}$, and $e^{i(2-\Omega) T_{0}}$ are capable of generating resonant behavior. Specifically, the following cases lead to the production of secular terms:

$$
\begin{array}{ll}
\Omega-2=1 & \rightarrow \Omega=3 \\
3 \Omega=1 & \rightarrow \Omega=1 / 3 \\
2-\Omega=1 ; 2 \Omega-1=1 ; \Omega=1 & \rightarrow \Omega=1
\end{array}
$$

The cases of $\Omega=3$ and $1 / 3$ correspond to the secondary resonances of the subharmonic and superharmonic type, respectively. In addition, $\Omega=1$ shows the case of primary hard excitation and is excluded, because the simultaneous presence of the hard excitation and primary resonance can be considered as a destructive case due to extremely large deflections. To characterize the energy harvester response under hard excitation comprehensively, the cases of the subharmonic and superharmonic resonances that are mentioned above will be investigated. 


\section{3-1- Subharmonic resonance}

First, the frequency response equation corresponding to the case of the subharmonic resonance $(\Omega \approx 3)$ will be obtained. To this end, it is assumed that $A=\frac{1}{2} a e^{i \beta}$, where $a$ and $\beta$ are real functions of $T_{1}$, and:

$$
\Omega=3+\varepsilon \sigma
$$

Here $\sigma$ is called the detuning parameter. Then, based on Eq. (13), the vanishing of the secular terms in this case requires that:

$$
-\left\{\left(a^{\prime}+a i \beta^{\prime}\right) i\right\}-\zeta a i-\frac{\Theta a i}{2(\alpha+i)}-\kappa\left\{\frac{3}{4} a^{2} F e^{i\left(\sigma T_{1}-3 \beta\right)}+\frac{3}{8} a^{3}+3 a F^{2}\right\}=0
$$

In addition, by defining $\gamma=\sigma T_{1}-3 \beta$ and separating the real and imaginary parts of Eq. (16), the electromechanical modulation equations are found as:

$$
\begin{aligned}
& a^{\prime}=-\zeta a-\frac{\Theta a \alpha}{2\left(\alpha^{2}+1\right)}-\frac{3}{4} a^{2} \kappa F \sin \gamma \\
& \gamma^{\prime}=\frac{3}{a}\left\{\frac{a \sigma}{3}-\frac{\Theta a}{2\left(\alpha^{2}+1\right)}-\kappa\left\{\frac{3}{8} a^{3}+3 a F^{2}\right\}-\frac{3}{4} a^{2} \kappa F \cos \gamma\right\}
\end{aligned}
$$

The steady-state form of Eq. (17) is obtained by setting $a^{\prime}=\gamma^{\prime}=0$ as:

$$
\begin{aligned}
& \frac{9}{4} a^{2} \kappa F \sin \gamma=-3\left(\zeta a+\frac{\Theta a \alpha}{2\left(\alpha^{2}+1\right)}\right) \\
& \frac{9}{4} a^{2} \kappa F \cos \gamma=\left\{\sigma a-\frac{3 \Theta a}{2\left(\alpha^{2}+1\right)}-\kappa\left\{\frac{9}{8} a^{3}+9 a F^{2}\right\}\right.
\end{aligned}
$$

By eliminating $\gamma$ from the relationships in Eq. (18), the frequency response curve of the displacement, $a$, in the subharmonic resonance can be stated as:

$$
\left[9\left(\zeta+\frac{\Theta \alpha}{2\left(\alpha^{2}+1\right)}\right)^{2}+\left(\sigma-\frac{3 \Theta}{2\left(\alpha^{2}+1\right)}-\kappa\left\{\frac{9}{8} a^{2}+9 F^{2}\right\}\right)^{2}\right]=\frac{81}{16} a^{2} \kappa^{2} F^{2}
$$

After solving Eq. (19) and obtaining the values of $a$, the voltage frequency response curve can be established by inserting the values of $a$ into Eq. (10) and calculating the maximum of its output, which will be denoted by $V$. 


\section{3-2- Superharmonic resonance}

Next, the frequency response equation corresponding to the superharmonic case $(\Omega \approx 1 / 3)$ will be obtained. In this scenario it is assumed that:

$$
3 \Omega=1+\varepsilon \sigma
$$

Based on Eq. (13), the disappearance of the secular terms in this case needs:

$$
-\left\{\left(a^{\prime}+a i \beta^{\prime}\right) i\right\}-\zeta a i-\frac{\Theta a i}{2(\alpha+i)}-\kappa\left\{\frac{3}{8} a^{3}+3 F^{2} a+F^{3} e^{i\left(\sigma T_{1}-\beta\right)}\right\}=0
$$

As for the previous case, by considering $\gamma=\sigma T_{1}-\beta$ and separating the real and imaginary parts of Eq. (21), the modulation equations are obtained as:

$$
\begin{aligned}
& a^{\prime}=-\zeta a-\frac{\Theta a \alpha}{2\left(\alpha^{2}+1\right)}-\kappa F^{3} \sin \gamma \\
& \gamma^{\prime}=\frac{1}{a}\left\{a \sigma-\frac{\Theta a}{2\left(\alpha^{2}+1\right)}-\kappa\left\{\frac{3}{8} a^{3}+3 a F^{2}\right\}-\kappa F^{3} \cos \gamma\right\}
\end{aligned}
$$

The steady-state form of Eq. (22) can be obtained by equating $a^{\prime}=\gamma^{\prime}=0$. These lead to:

$$
\begin{aligned}
& \sin \gamma=-\frac{1}{\kappa F^{3}}\left(\zeta a+\frac{\Theta a \alpha}{2\left(\alpha^{2}+1\right)}\right) \\
& \cos \gamma=\frac{1}{\kappa F^{3}}\left\{a \sigma-\frac{\Theta a}{2\left(\alpha^{2}+1\right)}-\kappa\left\{\frac{3}{8} a^{3}+3 a F^{2}\right\}\right\}
\end{aligned}
$$

Elimination of $\gamma$ between the relationships in Eq. (23) yields the frequency response equation of the superharmonic oscillations amplitude as:

$$
\left[\left(\zeta+\frac{\Theta \alpha}{2\left(\alpha^{2}+1\right)}\right)^{2}+\left(\sigma-\frac{\Theta}{2\left(\alpha^{2}+1\right)}-\kappa\left\{\frac{3}{8} a^{2}+3 F^{2}\right\}\right)^{2}\right] a^{2}=\left(\kappa F^{3}\right)^{2}
$$

The voltage frequency response curve in this case can also be constructed by introducing the values of $a$ that are obtained from Eq. (24) into Eq. (10).

Finally, it is worth providing the frequency equations for the soft primary resonance for further qualitative comparisons. When the amplitude of the external stimulus is soft, it can be said that $f=\varepsilon \bar{f}$. The 
frequency response equation corresponding to this case can be found using the previously mentioned procedures. The frequency response equations of the displacement amplitude $a$ can be written as [42]:

$$
\left\{\left(2 \zeta+\frac{\Theta \alpha}{\alpha^{2}+1}\right)^{2}+\left(\frac{3}{4} \alpha a^{2}+\frac{\Theta}{\alpha^{2}+1}-2 \sigma\right)^{2}\right\} a^{2}=4 \bar{f}^{2}
$$

Moreover, the frequency response equation of the voltage amplitude $V$ is:

$$
\left\{\left(2 \zeta+\frac{\Theta \alpha}{\alpha^{2}+1}\right)^{2}+\left(\frac{3}{4} \alpha a^{2}+\frac{\Theta}{\alpha^{2}+1}-2 \sigma\right)^{2}\right\}\left(\alpha^{2}+1\right) V^{2}=4 \bar{f}^{2}
$$

\section{4- Results and Discussions}

In this section the dynamical response of the electromechanical system will be examined utilizing MSMbased solutions and a numerical integration method. First, to verify the approximate-analytical MSMbased solutions, one can use numerical integration methods. For this purpose, to verify the approximate analytical solutions, the frequency response curves for particular cases will be plotted using MSM-based solutions and compared to numerical integration results. To solve the governing electromechanical equations using numerical integration methods, Eqs. (3a) and (3b) must be written in state-space form, based on the states:

$$
\left\{\begin{array}{l}
z_{1}=x \\
z_{2}=\dot{x} \\
z_{3}=v
\end{array}\right.
$$

hence, the state-space form of Eqs. (3a) and (3b) is written as:

$$
\begin{aligned}
& \dot{z}_{1}=z_{2} \\
& \dot{z}_{2}=-z_{1}-2 \zeta z_{2}-\kappa z_{1}^{3}+\Theta z_{3}+2 f \cos (\Omega t) \\
& \dot{z}_{3}=-z_{2}-\alpha z_{3}
\end{aligned}
$$

To provide a numerical solution for the above coupled system based on the numerical integration method, the fourth-order Runge-Kutta scheme will be used. Accordingly, in Fig. 2, the frequency response curves 
of the beam deflection for the cases of sub- and super-harmonic resonances along with soft primary resonance are plotted using the MSM solutions and the numerical integration method. Furthermore, the MSM- and numerical-based frequency response curves of the output voltage for the subharmonic, superharmonic, and soft primary resonance states are also plotted in Fig. 3. It must be mentioned that throughout this paper, the solid lines or dots represent the stable solutions and the dashed lines denote the unstable solutions. Of course the numerical approach will only produce the stable solutions.

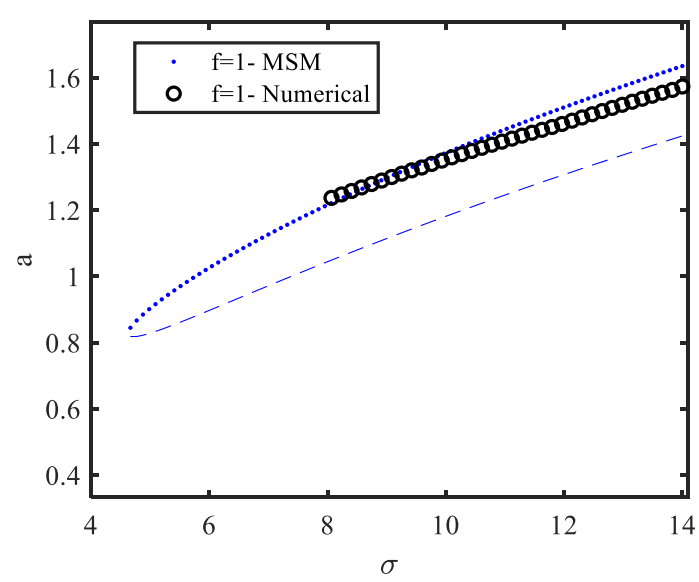

(a)

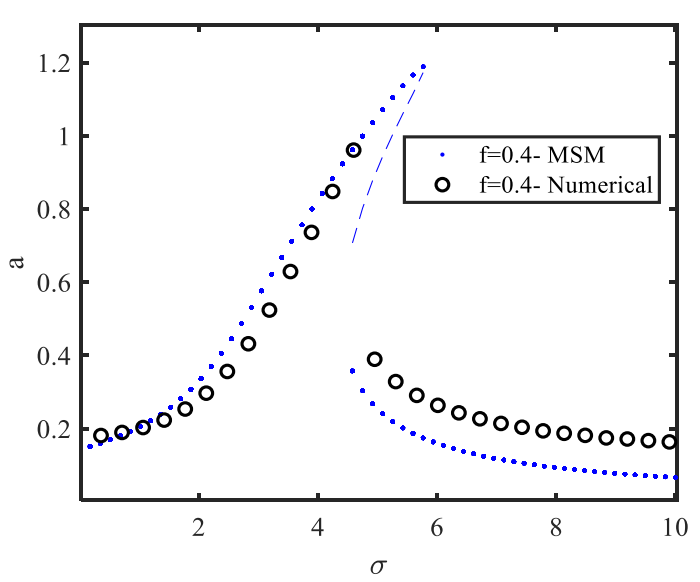

(b)

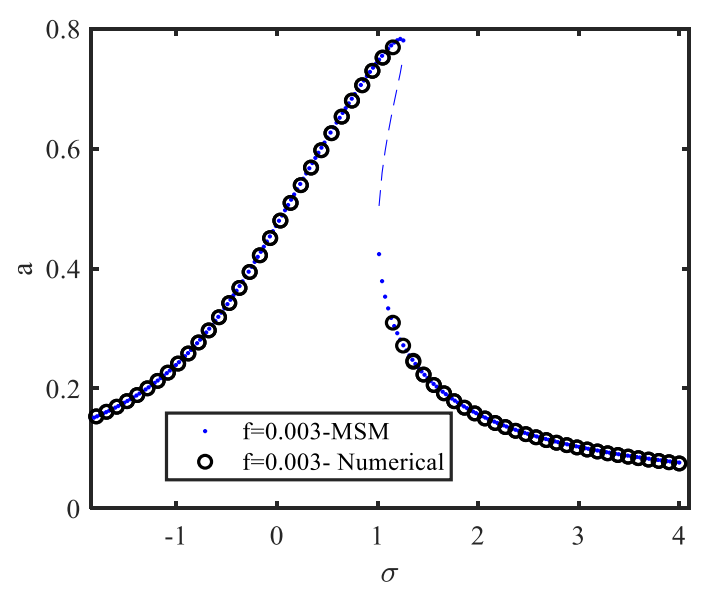

(c)

Fig. 2 Verification of the MSM-based frequency response curves of the deflection for (a) subharmonic, (b) superharmonic, and (c) soft primary resonance cases with the numerical integration method based on the fourth-order Runge-Kutta scheme 


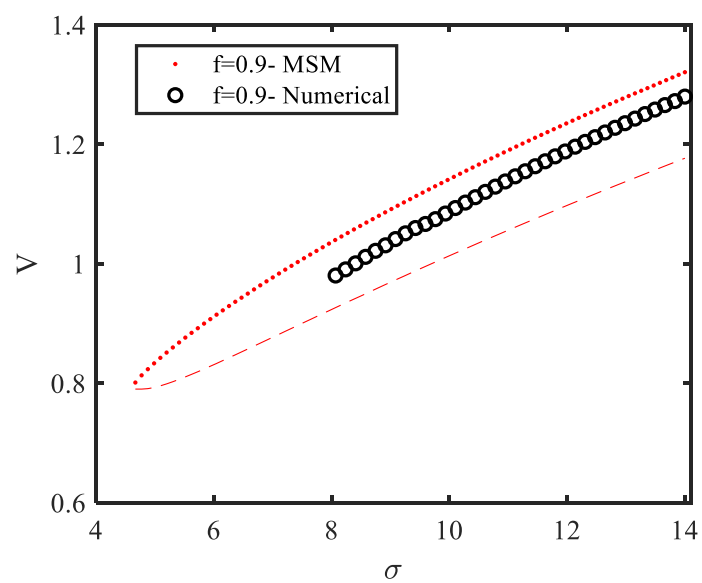

(a)

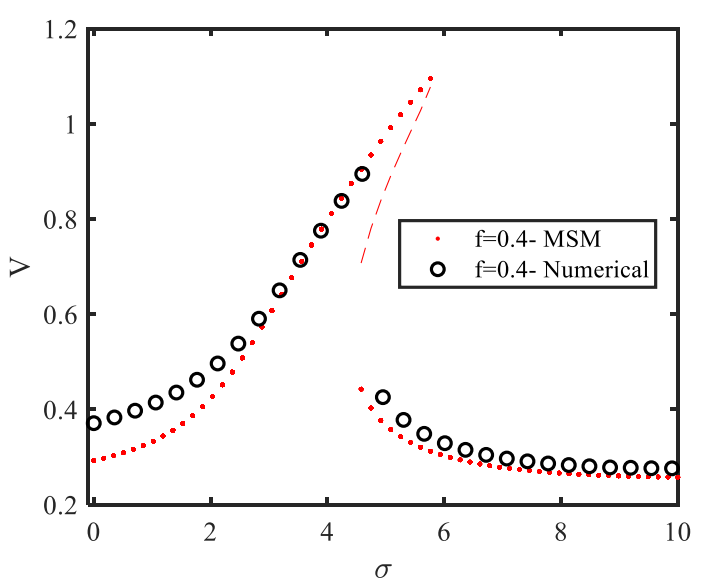

(b)

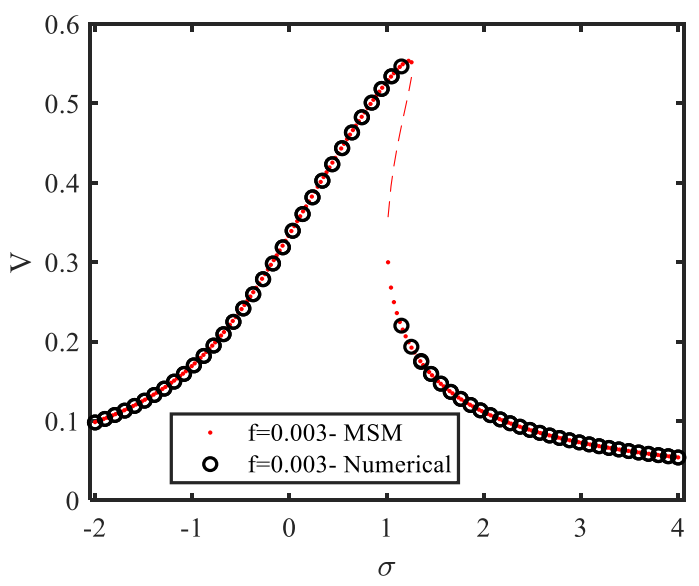

(c)

Fig. 3 Verification of the MSM-based frequency response curves of the output voltage for (a) subharmonic, (b) superharmonic, and (c) soft primary resonance scenarios with the numerical integration method based on the fourth-order Runge-Kutta scheme

As illustrated in Figs. 2 and 3, there is a good agreement between the MSM-based and numerical frequency response curves of the deflection and voltage for the three aforementioned cases. This implies that the approximate-analytical relationships and corresponding solutions are valid. However, based on Figs. 2a and $b$, a small difference can be observed between the MSM and the numerical deflection frequency response curves for the sub- and super-harmonic resonance cases, respectively. This is also true for the frequency response curves of the voltage in the sub-harmonic (Fig. 3a) and super-harmonic (Fig. 3a) states. These errors stem from the fact that the numerical integration scheme solves a differential 
equation and includes all of the resonance points of the electromechanical system. Hence at each resonance point, the frequency response curve is slightly affected by the frequency response curves of the lower and higher resonance frequencies. This difference was also shown in an experimentally validated study by Abusoua and Daqaq [1].

Next, qualitative comparisons between the secondary resonances and primary resonance cases are given. First, the frequency response curves of the dimensionless displacement and voltage for the primary resonance case for different forcing levels are given in Fig. 4, using Eqs. (25) and (26). In what follows, the dimensionless time constant ratio is assumed to be one $(\alpha=1)$.

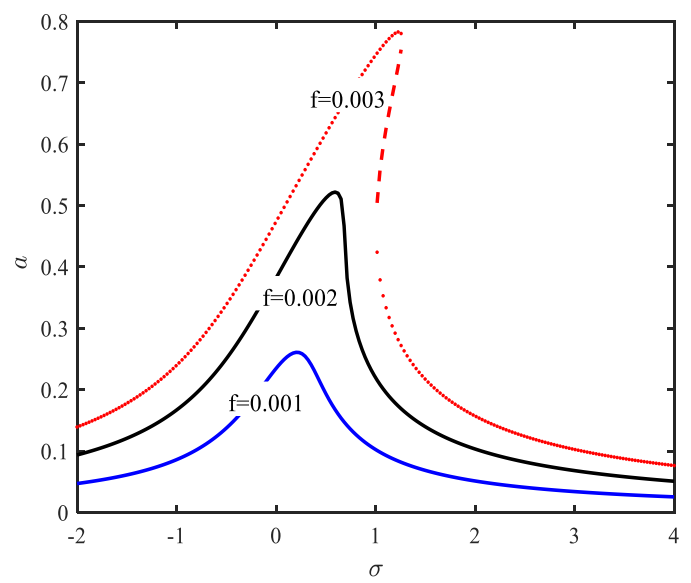

(a)

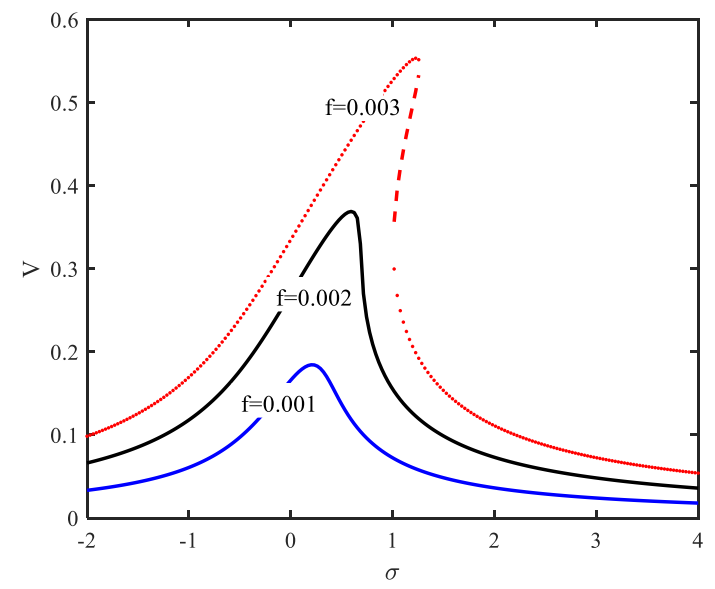

(b)

Fig. 4 Soft primary resonance frequency response curves of dimensionless (a) displacement and (b) voltage for different excitation levels

As illustrated in Fig. 4, the displacement and generated voltage are both amplified by increasing the excitation level, $f$. Moreover, as shown by Figs. $4 a$ and $b$, by increasing the excitation amplitude, the response behavior changes from linear to nonlinear hardening due to the presence of the positive cubic stiffness term in the equations. This causes the frequency response curves bend to the right and demonstrate jump phenomena, which yields a multivalued solution. Hence, in the vicinity of the resonance point, the initial conditions determine the branch to which the response attracts. However, for energy harvesting purposes, the initial conditions must be chosen such that the response follows the high amplitude, and consequently, high voltage branches. Moreover, as shown in Figs. 4a and b, the nonlinear behavior is intensified by increasing the excitation level. In addition, the force response curves of the 
displacement and voltage in the primary resonance case are shown in Figs. 5a and b, respectively, considering three different detuning parameters and $f=0.003$.

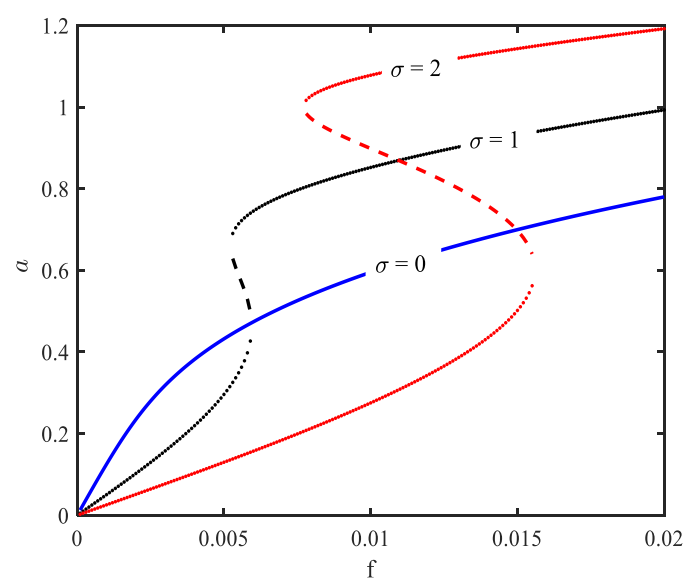

(a)

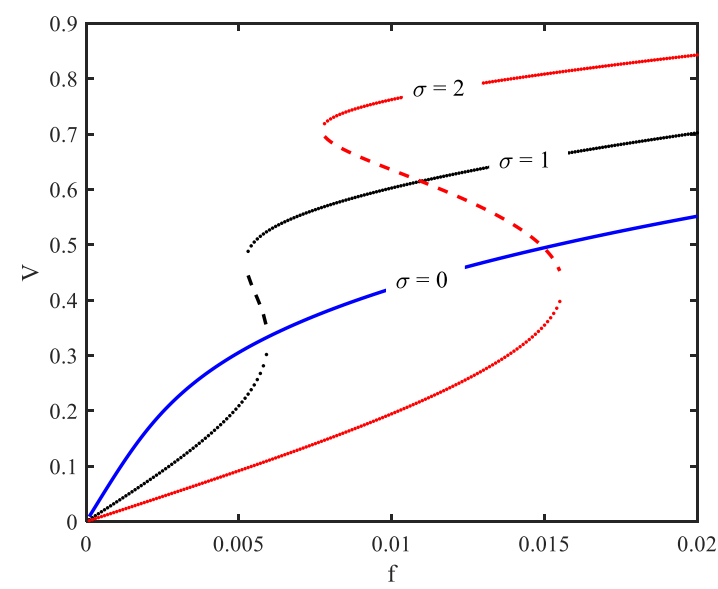

(b)

Fig. 5 Primary resonance force response curves of dimensionless (a) displacement and (b) voltage for different detuning parameters

Figures $5 \mathrm{a}$ and $\mathrm{b}$ show the responses of the displacement and voltage for different detuning parameter values, and also exhibit jump phenomena and multivalued solutions. As stated previously, the initial conditions determine the branch which the solutions follow.

Now, to characterize the response of the PZT energy harvester in the cases of the subharmonic and superharmonic resonances, the level of the external forcing must be increased. First, using Eqs. (19) and (10), the frequency response curves of the displacement and voltage in the case of subharmonic resonance are given in Fig. 6, considering three different forcing levels. 


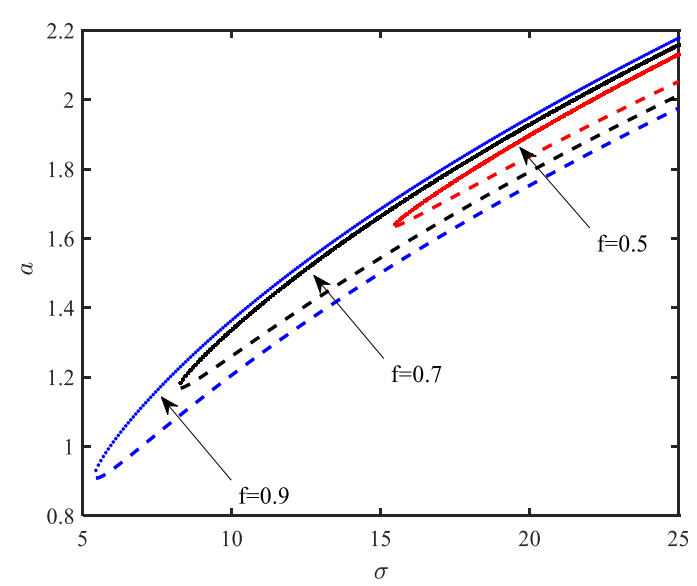

(a)

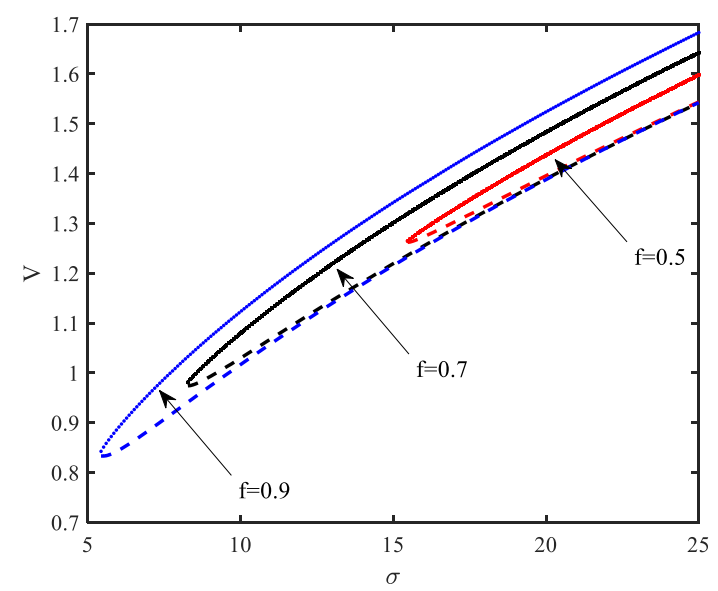

(b)

Fig. 6 Subharmonic resonance frequency response curves of dimensionless (a) displacement and (b) voltage for different excitation levels

According to Fig. 6, the general behavior of the frequency response curves differ from the graphs reported in Fig. 4. Furthermore, although the frequency of the excitation is three times the fundamental frequency of the system $(\Omega \approx 3)$, the bimorph experiences considerably larger amplitude oscillations compared to the primary resonance case reported in Fig. 4a. This originates from the hard forcing amplitude. Hence, as shown in Fig. 6b, the energy harvester produces significantly higher voltages compared to the primary resonance scenario, i.e. Fig. 4b. This also implies that the harvester resonance bandwidth is extended towards the larger frequencies, rendering a broadband PVEH. Moreover, as is clear from Figs. 6a and b, by increasing the forcing amplitude, the harvester starts to oscillate and generate voltage for smaller detuning parameters. Thus, for harder excitations, the system vibrates and delivers electrical output at smaller frequencies. This indicates that the subharmonic resonance regions are very sensitive to the excitation amplitude, which was also stated in Ref [45]. In addition, although the stable and unstable responses coexist in Fig. 6, no jump phenomenon occurs and the initial conditions determine the solution branch. The dimensionless deflection and voltage force response curves of the subharmonic resonance are illustrated in Fig. 7, for three different detuning parameters values and $\alpha=3$. 


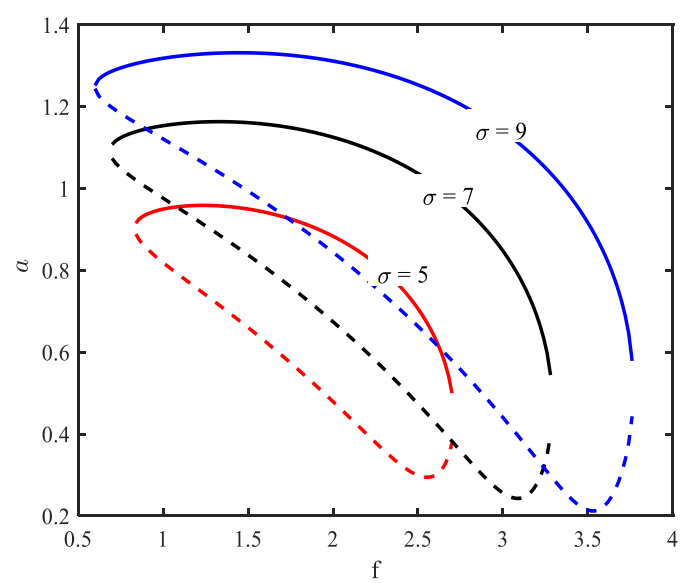

(a)

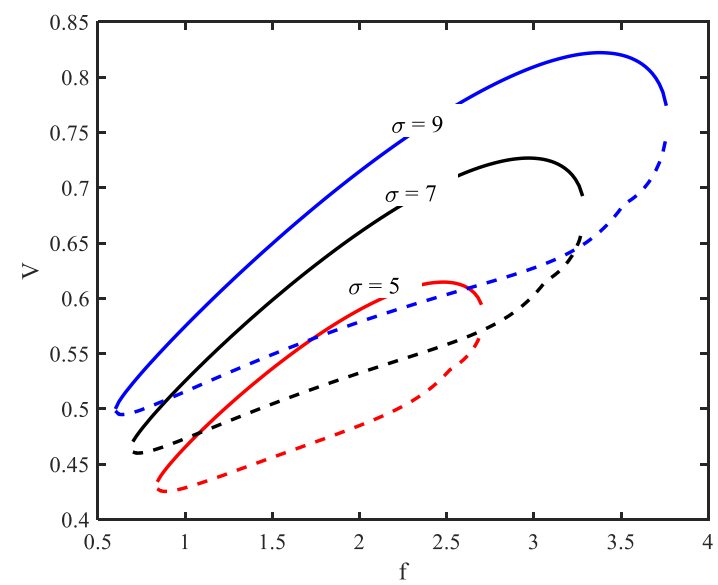

(b)

Fig. 7 Subharmonic resonance force response curves of dimensionless (a) displacement and (b) voltage for different detuning parameters

Figures $7 \mathrm{a}$ and $\mathrm{b}$ reveal that as the excitation amplitude increases, the resonance region expands and the energy harvester can experience resonant oscillations and consequently generate high amplitude voltages over a larger frequency range. Moreover, although there are multivalued solutions in the displacement and voltage force response curves, the jump phenomenon does not take place and the initial conditions dictate the response value. Next, to examine the dynamical response of the PVEH in the superharmonic resonance case $(\Omega \approx 1 / 3)$, the corresponding frequency response curves of the displacement and voltage for different excitation amplitudes are plotted in Figs 8a and b, respectively, using Eqs. (24) and (10). Inspecting Fig. 8, the first thing that draws attention is that, contrary to the subharmonic scenario, the behavior of the deflection and voltage frequency response curves resembles the primary resonance case. Specifically, the frequency response curves are of the hardening type and there are jump phenomena and multivalued solutions in the subharmonic case. Moreover, as illustrated in Fig. 8a, in this case, the deflection is considerably larger than the primary resonance response (Fig. 4a). This originates from the hard nature of the excitation. Subsequently, as revealed by Fig. 8b, the output voltage is also amplified. Specifically, by inspecting Fig. 8b, it is clear that, the level of the generated voltage is boosted considerably compared to the case of the primary resonance that is reported in Fig. 4b. 


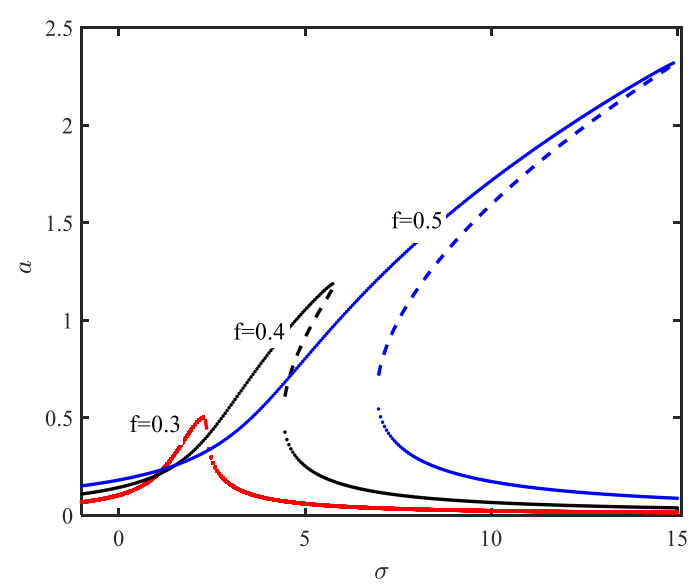

(a)

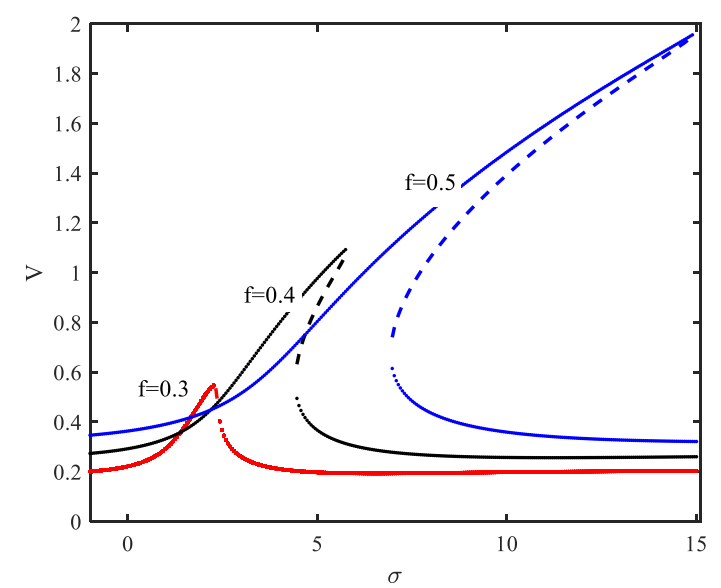

(b)

Fig. 8 Superharmonic resonance frequency response curves of dimensionless (a) displacement and (b) voltage for different excitation levels

This implies that the PVEH performs efficiently in the case of superharmonic resonance. Hence, the resonance bandwidth increases towards the lower frequencies, resulting in wideband energy capture. The displacement and voltage force response curves for the superharmonic case are shown in Fig. 9.

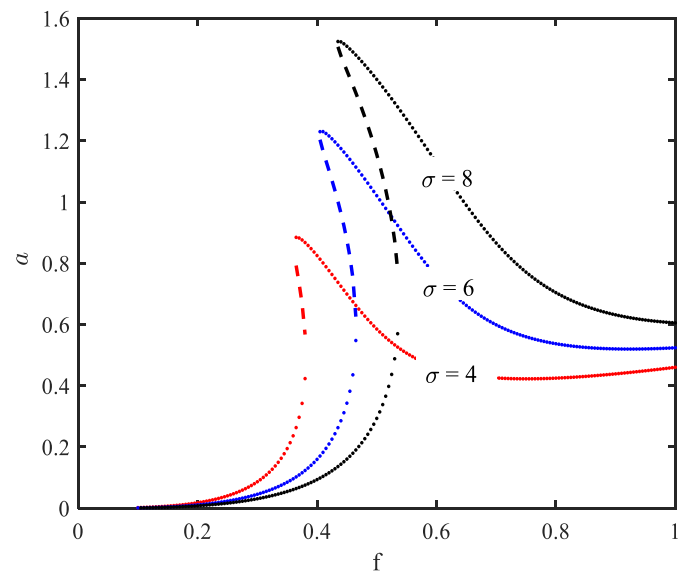

(a)

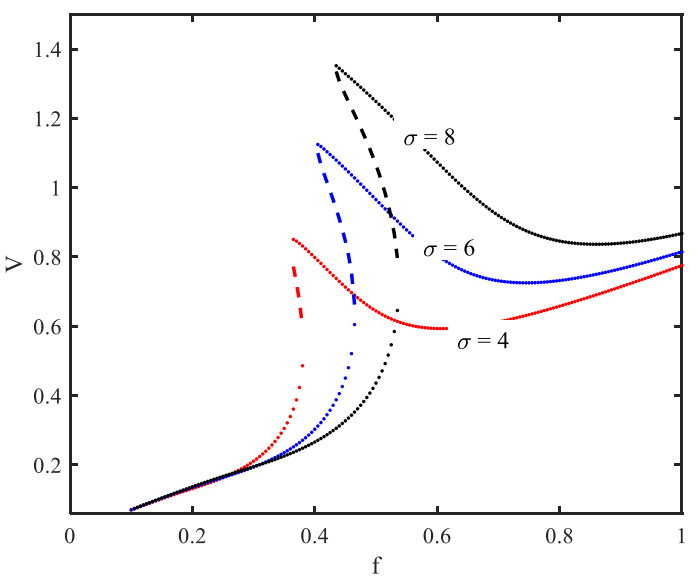

(b)

Fig. 9 Superharmonic resonance force response curves of dimensionless (a) displacement and (b) voltage for different detuning parameters

In contrast to Fig. 8, in which the frequency response curves of the superharmonic case are similar to the primary resonance case, Fig. 9 shows that there is an essential difference between the force response 
curves of the superharmonic and primary resonance scenarios. Precisely, based on Fig. 9, as the forcing amplitude increases, the deflection and voltage response curves experience a jump at the bifurcation point. Subsequently, the responses start to decrease steeply with further increases in the forcing amplitude up to a specific point, after which, the amplitude increases. In contrast, considering Fig. 5, in the primary resonance state, the responses undergo a monotonic increase after the upward jump occurs.

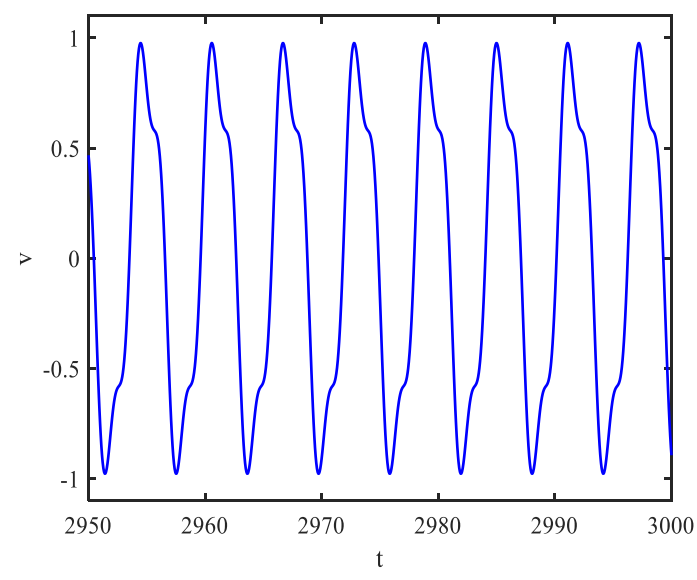

(a)

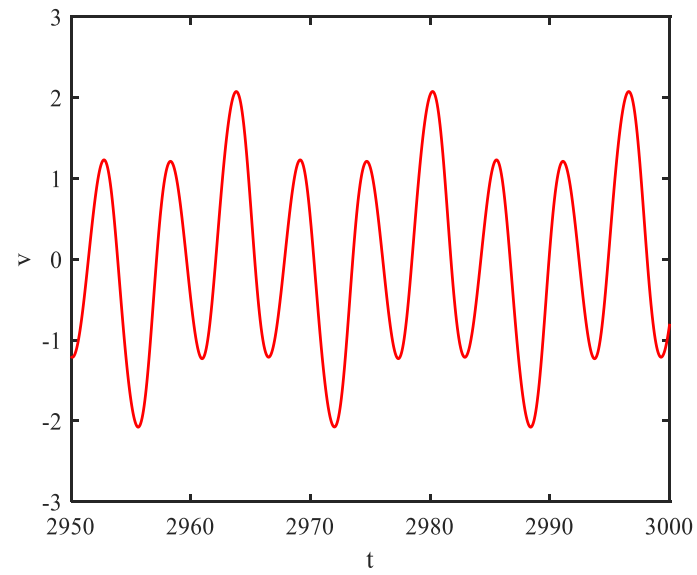

(c)

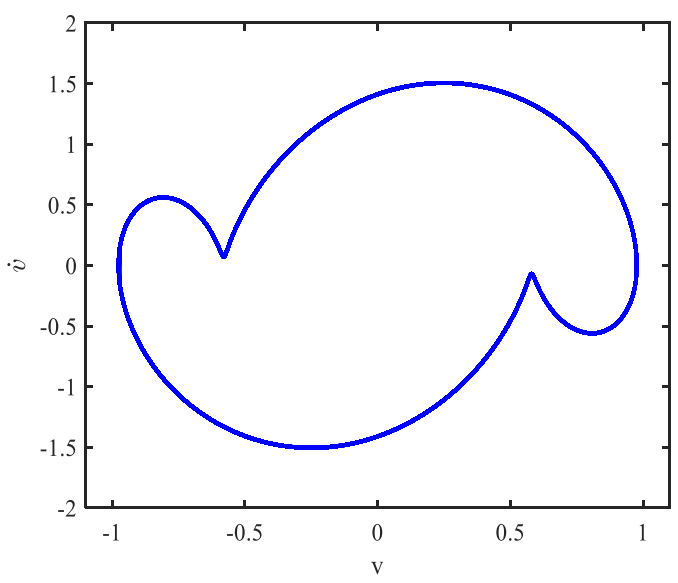

(b)

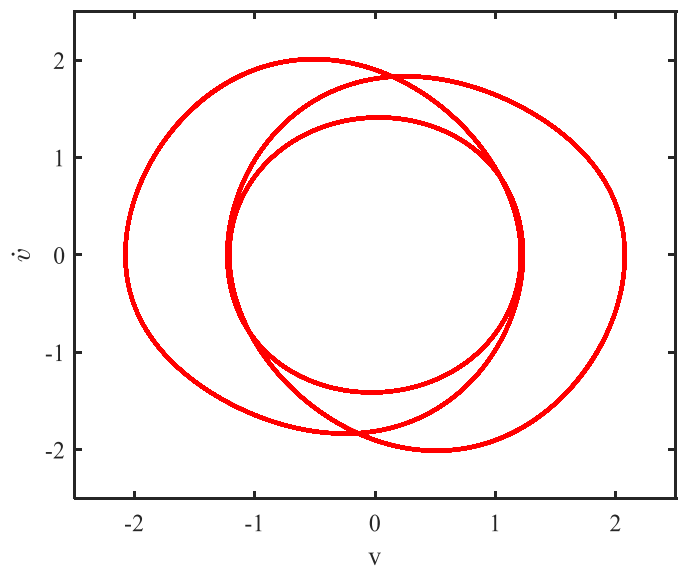

(d)

Fig. 10 (a) The time history and (b) phase plane of the voltage for the subharmonic case ; (c) the time history and (d) phase plane of the voltage for the superharmonic case with $f=0.9$

Next, utilizing the numerical integration method, one can examine the time-domain response of the systems in the secondary resonances. Hence, the time histories and phase-plane responses of the voltage in the sub- and super-harmonic resonance cases are plotted in Fig. 10, for $f=0.9$. According to Figs. $10 a$ 
and $c$, the level of the harvested voltage for the case of the superharmonic resonance is larger than that of the subharmonic. This can also be observed in the phase diagram of Figs. $10 \mathrm{~b}$ and $\mathrm{d}$. Specifically, the trajectories in the phase planes reveal that the voltage in superharmonic scenario is larger compared to the subharmonic resonance.

Up to this point, the study has concentrated on the energy harvester amplitude and voltage responses under hard excitation. However, for energy harnessing purposes, the most important criterion that determines the efficiency of any newly proposed system is its maximum or optimum electrical output power. Consequently, the optimum power is required. In linear systems there is an analytical relationship that provides the maximum power and the corresponding optimum time constant ratio as a function of the energy harvester parameters [46]. The considered PVEH is a nonlinear system for which no closedform solution can be found in general. Hence, finding an analytical solution for the maximum power is not possible. However, to get an insight into the optimum electrical output, examining the variations of the output power versus the time constant ratio and detuning parameter can be informative, because, the output power can be assessed against two prominent and determinative parameters. The dimensionless output power can be defined, using Eq. (2) and (4), as [47]:

$$
P=\alpha V^{2}
$$

Using Eq. (29) and the previously obtained voltage frequency response equations, the three dimensional graphs of the output power versus the time constant ratio and detuning parameter for the sub- and superharmonic cases are given in Figs. 11a and b, respectively. To construct Fig. 11a, the frequency response curves of the output power versus the detuning parameter are plotted for various time constant ratios, using Eqs. (10), (19), and (29). Furthermore, Eqs. (10), (24), and (29) are utilized to establish the frequency response curves of the power in Fig. 11b. Here, the forcing amplitude is assumed to be 0.5 and the detuning parameter is in the range of $0 \leq \sigma \leq 30$. Figure 11 clearly shows that, for each case, changing the time constant ratio can alter the quantitative behavior of the response, not the qualitative behavior. In addition, as Figs. 11a and $b$ show, by changing the time constant ratio at each detuning parameter, the output power reaches a maximum value. Hence, for a specific detuning parameter and forcing level, there exists an optimum power and associated time constant ratio. 
To obtain the optimum output power and accompanying time constant ratio for the considered cases concisely, one can utilize Fig. 11. By extracting the precise values of the maximum output power and corresponding values of $\alpha$ from the graphs represented in Figs. 11a and $\mathrm{b}$ and plotting them, one can obtain Fig. 12. Hence, Fig. 12 illustrates the optimum or maximum output power and the corresponding time constant ratio for each of the aforementioned scenarios.

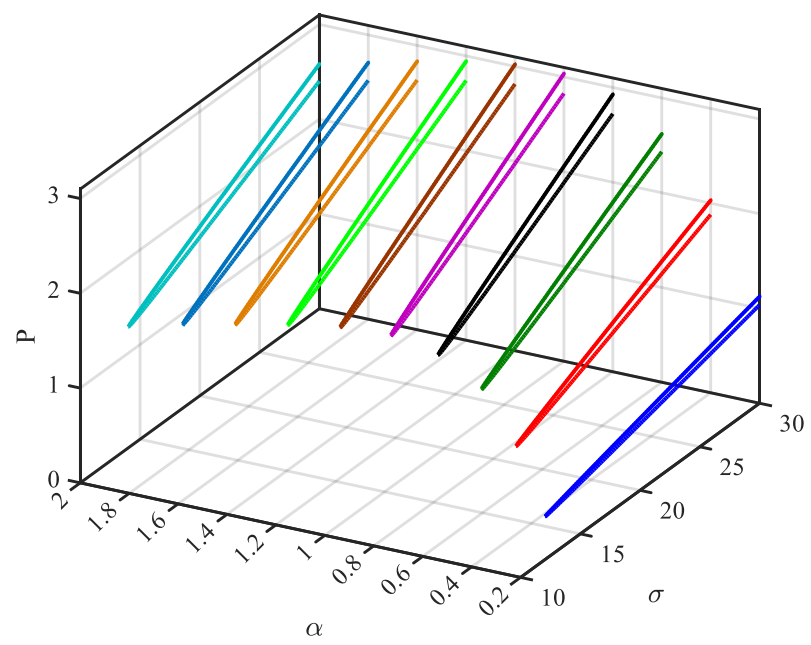

(a)

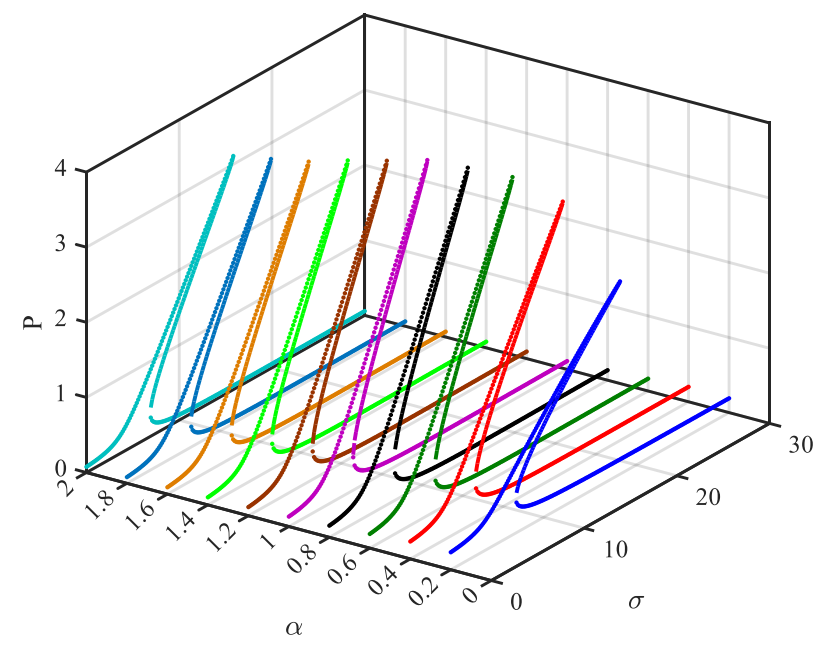

(b)

Fig. 11 Three dimensional graphs of the output power versus the time constant ratio and detuning parameter for (a) subharmonic (b) superharmonic resonance cases

Based on to Fig. 11, in the considered range of the detuning parameter, the PVEH produces larger power for the superharmonic resonance case, compared to the subharmonic resonance state. Specifically, the maximum dimensionless powers for the super- and sub-harmonic cases are 3.9 and 3, respectively. This implies that the optimum power corresponding to the superharmonic case is 30 percent higher than the subharmonic scenario. The optimum output powers of the sub- and super-harmonic scenarios along with relevant time constant ratios for two other forcing levels of $f=0.55$ and 0.6 are also evaluated using the same approach and the results are reported in Table 2. 


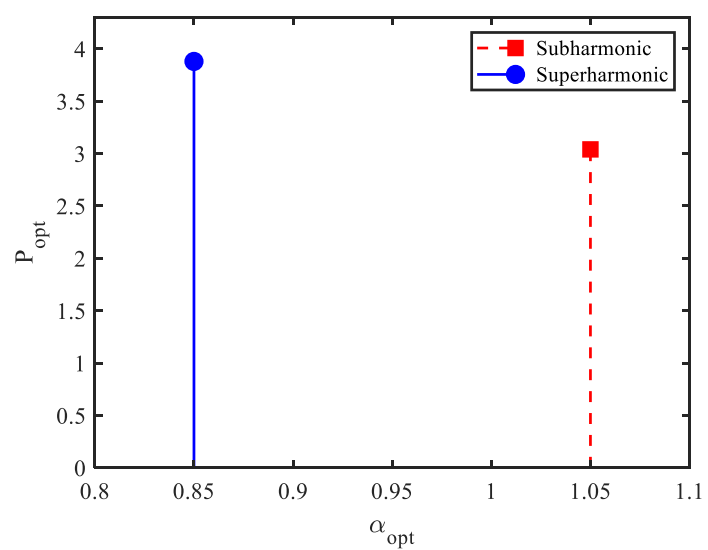

Fig. 12 Optimum electrical power and the corresponding time constant ratio for the subharmonic and superharmonic cases

Table 2. The optimum power of the sub- and super-harmonic scenarios for some various forcing amplitudes

\begin{tabular}{cccc}
\hline \hline Forcing level & Subharmonic & Superharmonic & Difference \\
\hline \hline$f=0.5$ & 3 & 3.9 & $30 \%$ \\
$f=0.55$ & 3.08 & 5.24 & $70 \%$ \\
$f=0.6$ & 3.12 & 5.98 & $91 \%$ \\
\hline \hline
\end{tabular}

Based on Table 2, also for the forcing levels of $f=0.55$ and 0.6 , the output power in the superharmonic resonance state outweighs the power of the subharmonic scenario. Thus, the maximum harvested power in the former case is respectively 70 and 91 percent higher compared to the latter case. for $f=0.55$ and $f=0.6$, respectively.

\section{4-Conclusions}

In this article, energy harvesting from the secondary resonances of a nonlinear PZT beam under a hard periodic excitation is investigated. The system comprises a cantilever unimorph PZT beam with an attached tip mass and is exposed to an harmonic tip force. First, the dimensionless ordinary differential equations (ODEs) governing the electromechanical system are obtained. Then, the multiple scales method (MSM) is applied to provide an approximate-analytical solution for the ODEs. Primarily, the MSM solutions 
showed that the hard stimulus results in secondary resonances of the sub- and super-harmonic types. The numerical integration method was then utilized to verify the MSM-based solutions. The results show that there is a good agreement between the numerical and approximate-analytical solutions. Next, to characterize the dynamical response of the system, the frequency and force response curves of the deflection and voltage for the cases of the subharmonic, superharmonic, and soft primary resonances are plotted using the MSM-based solutions. The results revealed that the generated voltages in the sub-and super-harmonic resonance scenarios are significantly higher, rendering the non-resonant hard excitation as a superb choice for energy harvesting. Moreover, this implies that the resonance bandwidth is broadened towards the lower and upper portions of the primary resonance vicinity in the superharmonic and subharmonic cases, respectively. The time histories of the output voltage and the phase planes revealed that the level of the generated voltage in the superharmonic scenario is larger than the subharmonic case. Finally, to assess the output power of the system in the various secondary resonances, the electrical power of the system is studied for different detuning parameters and time constant ratios. This investigation revealed that the optimum dimensionless power for the superharmonic resonance is larger than for the subharmonic resonance. Specifically, comparing the output powers, it was shown that, at the forcing levels of $0.5,0.55$, and 0.6 , the output power corresponding to the superharmonic resonance is respectively 30,70 , and 91 percent higher than the subharmonic case.

\section{Declarations}

Funding: This study was funded by Tarbiat Modares University (IG-39703).

Conflicts of interest/Competing interests: The authors declare that they have no conflict of interest.

Availability of data and material: Not applicable

Code availability: Not applicable 


\section{References}

[1] A. Syta, C. R. Bowen, H. A. Kim, A. Rysak, and G. Litak, "Experimental analysis of the dynamical response of energy harvesting devices based on bistable laminated plates," Meccanica, journal article vol. 50, no. 8, pp. 1961-1970, August 012015.

[2] Q. He and M. F. Daqaq, "Electric load optimization of a nonlinear mono-stable duffing harvester excited by white noise," Meccanica, journal article vol. 51, no. 5, pp. 1027-1039, May 012016.

[3] G. Litak, M. I. Friswell, and S. Adhikari, "Regular and chaotic vibration in a piezoelectric energy harvester," Meccanica, journal article vol. 51, no. 5, pp. 1017-1025, May 012016.

[4] S. P. Beeby et al., "A micro electromagnetic generator for vibration energy harvesting," Journal of Micromechanics and microengineering, vol. 17, no. 7, p. 1257, 2007.

[5] A. B. Rostami and M. Armandei, "Renewable energy harvesting by vortex-induced motions: Review and benchmarking of technologies," Renewable and Sustainable Energy Reviews, vol. 70, pp. 193-214, 2017.

[6] A. Abdelkefi, "Aeroelastic energy harvesting: A review," International Journal of Engineering Science, vol. 100, pp. 112-135, 2016.

[7] M. F. Daqaq et al., "Micropower Generation Using Cross-Flow Instabilities: A Review of the Literature and Its Implications," Journal of Vibration and Acoustics, vol. 141, no. 3, p. 030801, 2019.

[8] M. Rezaei and R. Talebitooti, "Wideband PZT Energy Harvesting from the Wake of a Bluff Body in Varying Flow Speeds," International Journal of Mechanical Sciences, p. 105135, 2019/09/05/ 2019.

[9] S. Liu, P. Li, and Y. Yang, "On the design of an electromagnetic aeroelastic energy harvester from nonlinear flutter," Meccanica, journal article vol. 53, no. 11, pp. 2807-2831, September 012018.

[10] J. Silva-Leon, A. Cioncolini, M. R. Nabawy, A. Revell, and A. Kennaugh, "Simultaneous wind and solar energy harvesting with inverted flags," Applied Energy, vol. 239, pp. 846-858, 2019.

[11] Y. Suzuki, D. Miki, M. Edamoto, and M. Honzumi, "A MEMS electret generator with electrostatic levitation for vibration-driven energy-harvesting applications," Journal of Micromechanics and Microengineering, vol. 20, no. 10, p. 104002, 2010.

[12] M. Lallart, S. Pruvost, and D. Guyomar, "Electrostatic energy harvesting enhancement using variable equivalent permittivity," Physics Letters A, vol. 375, no. 45, pp. 3921-3924, 2011.

[13] Z. Yang, Y. Tan, and J. Zu, "A multi-impact frequency up-converted magnetostrictive transducer for harvesting energy from finger tapping," International Journal of Mechanical Sciences, vol. 126, pp. 235-241, 2017.

[14] R. Naseer, H. Dai, A. Abdelkefi, and L. Wang, "Piezomagnetoelastic energy harvesting from vortex-induced vibrations using monostable characteristics," Applied Energy, vol. 203, pp. 142153, 2017.

[15] K. Fan, Y. Zhang, H. Liu, M. Cai, and Q. Tan, "A nonlinear two-degree-of-freedom electromagnetic energy harvester for ultra-low frequency vibrations and human body motions," Renewable Energy, vol. 138, pp. 292-302, 2019.

[16] D. Castagnetti, "A simply tunable electromagnetic pendulum energy harvester," Meccanica, journal article vol. 54, no. 6, pp. 749-760, April 012019.

[17] M. Rezaei, S. E. Khadem, and P. Firoozy, "Broadband and tunable PZT energy harvesting utilizing local nonlinearity and tip mass effects," International Journal of Engineering Science, vol. 118, pp. 1-15, 2017. 
[18] M. Rezaei, R. Talebitooti, and M. I. Friswell, "Efficient acoustic energy harvesting by deploying magnetic restoring force," Smart Materials and Structures, 2019.

[19] D. Castagnetti and E. Radi, "A piezoelectric based energy harvester with dynamic magnification: modelling, design and experimental assessment," Meccanica, journal article vol. 53, no. 11, pp. 2725-2742, September 012018.

[20] A. Erturk and D. J. Inman, "A distributed parameter electromechanical model for cantilevered piezoelectric energy harvesters," Journal of vibration and acoustics, vol. 130, no. 4, p. 041002, 2008.

[21] A. Erturk and D. J. Inman, "An experimentally validated bimorph cantilever model for piezoelectric energy harvesting from base excitations," Smart materials and structures, vol. 18, no. 2, p. 025009, 2009.

[22] S. C. Stanton, C. C. McGehee, and B. P. Mann, "Nonlinear dynamics for broadband energy harvesting: Investigation of a bistable piezoelectric inertial generator," Physica D: Nonlinear Phenomena, vol. 239, no. 10, pp. 640-653, 2010.

[23] S. Dhote, Z. Yang, K. Behdinan, and J. Zu, "Enhanced broadband multi-mode compliant orthoplanar spring piezoelectric vibration energy harvester using magnetic force," International Journal of Mechanical Sciences, vol. 135, pp. 63-71, 2018.

[24] S. Roundy and Y. Zhang, "Toward self-tuning adaptive vibration-based microgenerators," in Smart Structures, Devices, and Systems II, 2005, vol. 5649, pp. 373-385: International Society for Optics and Photonics.

[25] W.-J. Wu, Y.-Y. Chen, B.-S. Lee, J.-J. He, and Y.-T. Peng, "Tunable resonant frequency power harvesting devices," in Smart Structures and Materials 2006: Damping and Isolation, 2006, vol. 6169, p. 61690A: International Society for Optics and Photonics.

[26] S.-C. Huang and C.-Y. Tsai, "Theoretical analysis of a new adjustable broadband PZT beam vibration energy harvester," International Journal of Mechanical Sciences, vol. 105, pp. 304-314, 2016.

[27] P. Alevras, S. Theodossiades, and H. Rahnejat, "On the dynamics of a nonlinear energy harvester with multiple resonant zones," Nonlinear Dynamics, pp. 1-16, 2018.

[28] M. F. Daqaq, R. Masana, A. Erturk, and D. D. Quinn, "On the role of nonlinearities in vibratory energy harvesting: a critical review and discussion," Applied Mechanics Reviews, vol. 66, no. 4, p. 040801, 2014.

[29] H. Deng et al., "Poly-stable energy harvesting based on synergetic multistable vibration," Communications Physics, vol. 2, no. 1, p. 21, 2019.

[30] R. Masana and M. F. Daqaq, "Energy harvesting in the super-harmonic frequency region of a twin-well oscillator," Journal of Applied Physics, vol. 111, no. 4, p. 044501, 2012.

[31] J.-T. Lin, K. Walsh, and B. Alphenaar, "Enhanced stochastic, subharmonic, and ultraharmonic energy harvesting," Journal of Intelligent Material Systems and Structures, vol. 24, no. 11, pp. 1324-1331, 2013.

[32] A. Syta, G. Litak, M. I. Friswell, and S. Adhikari, "Multiple solutions and corresponding power output of a nonlinear bistable piezoelectric energy harvester," The European Physical Journal B, vol. 89, no. 4, p. 99, 2016.

[33] T. Huguet, A. Badel, and M. Lallart, "Exploiting bistable oscillator subharmonics for magnified broadband vibration energy harvesting," Applied Physics Letters, vol. 111, no. 17, p. 173905, 2017.

[34] M. Panyam, M. F. Daqaq, and S. A. Emam, "Exploiting the subharmonic parametric resonances of a buckled beam for vibratory energy harvesting," Meccanica, journal article vol. 53, no. 14, pp. 3545-3564, November 012018. 
[35] T. Huguet, A. Badel, O. Druet, and M. Lallart, "Drastic bandwidth enhancement of bistable energy harvesters: Study of subharmonic behaviors and their stability robustness," Applied energy, vol. 226, pp. 607-617, 2018.

[36] L. Chen, S. Pan, Y. Fei, W. Zhang, and F. Yang, "Theoretical study of micro/nano-scale bistable plate for flexoelectric energy harvesting," Applied Physics A, vol. 125, no. 4, p. 242, 2019.

[37] A. H. Nayfeh and D. T. Mook, Nonlinear oscillations. John Wiley \& Sons, 2008.

[38] A. Abusoua and M. F. Daqaq, "On using a strong high-frequency excitation for parametric identification of nonlinear systems," Journal of Vibration and Acoustics, vol. 139, no. 5, p. 051012, 2017.

[39] A. Abusoua and M. F. Daqaq, "Changing the nonlinear resonant response of an asymmetric mono-stable oscillator by injecting a hard high-frequency harmonic excitation," Journal of Sound and Vibration, vol. 436, pp. 262-272, 2018.

[40] M. F. Daqaq, "Characterizing the response of galloping energy harvesters using actual wind statistics," Journal of Sound and Vibration, vol. 357, pp. 365-376, 2015.

[41] A. Bibo, A. H. Alhadidi, and M. F. Daqaq, "Exploiting a nonlinear restoring force to improve the performance of flow energy harvesters," Journal of Applied Physics, vol. 117, no. 4, p. 045103, 2015.

[42] A. Erturk and D. J. Inman, Piezoelectric energy harvesting. John Wiley \& Sons, 2011.

[43] A. H. Nayfeh, Perturbation methods. John Wiley \& Sons, 2008.

[44] M. Rezaei, R. Talebitooti, and S. Rahmanian, "Efficient energy harvesting from nonlinear vibrations of PZT beam under simultaneous resonances," (in English), Energy, Article vol. 182, pp. 369-380, 2019.

[45] J. Peng, M. Xiang, L. Li, H. Sun, and X. Wang, "Time-Delayed Feedback Control of Piezoelectric Elastic Beams under Superharmonic and Subharmonic Excitations," Applied Sciences, vol. 9, no. 8, p. 1557, 2019.

[46] N. Elvin and A. Erturk, Advances in energy harvesting methods. Springer Science \& Business Media, 2013.

[47] S. C. Stanton, B. A. Owens, and B. P. Mann, "Harmonic balance analysis of the bistable piezoelectric inertial generator," Journal of Sound and Vibration, vol. 331, no. 15, pp. 3617-3627, 2012. 